\title{
Block Copolymer Micelles Generated by Crystallization-Driven Self-Assembly in Polymer Matrices
}

\author{
Jessica Gutiérrez González, ${ }^{1}$ Ruth N. Schmarsow, ${ }^{1}$ Úrsula M. Montoya Rojo, ${ }^{2,3}$ \\ Julieta Puig, ${ }^{1}$ Walter F. Schroeder, ${ }^{1}$ and Ileana A. Zucchii ${ }^{1 *}$ \\ ${ }^{1}$ Instituto de Investigaciones en Ciencia y Tecnología de Materiales (INTEMA), Universidad Nacional de Mar del Plata (UNMdP)-Conse- \\ jo Nacional de Investigaciones Científicas y Técnicas (CONICET), Argentina. \\ ${ }^{2}$ Grupo de Biotecnología y Biosíntesis. Instituto de Tecnología en Polímeros y Nanotecnología (ITPN-UBA-CONICET), Facultad de \\ Ingeniería, Universidad de Buenos Aires, Argentina \\ ${ }^{3}$ Departamento de Tecnología Industrial, Facultad de Ingeniería, Universidad de Buenos Aires, Argentina \\ * Corresponding author. E-mail: ileanazu@yahoo.com.ar
}

\begin{abstract}
In this review, we show how Crystallization-Driven Self-Assembly (CDSA), a method originally employed for the self-assembly of block copolymers in solution, was extended to the synthesis of elongated micellar nanostructures in polymer matrices. By highlighting some of the works published by our group in this area, the conditions to synthesize nanostructured polymers by CDSA are discussed. The knowledge of these conditions will allow developing a new generation of nanomaterials with tailored architecture based on a given application.
\end{abstract}

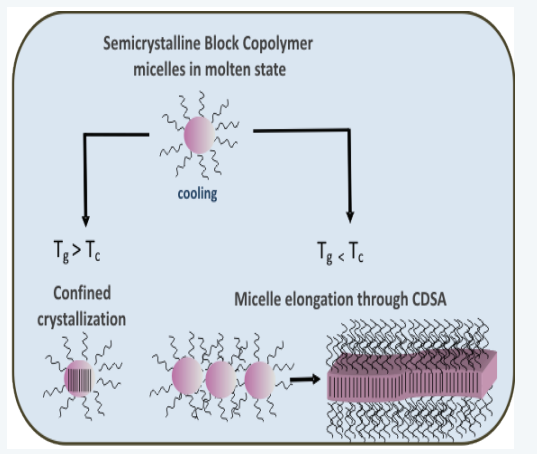

\section{Keywords:}

block copolymer, epoxy, micelles, nanoribbons, self-assembly, vesicles

\section{Introduction}

The ability of block copolymers (BCP) to form a variety of nanoscale periodic patterns through self-assembly offers a powerful tool for the 'bottom-up' fabrication of nanostructures with different levels of ordering. A BCP consists of two or more polymeric chains (blocks), which are chemically different and covalently attached to each other. If the constituent polymers are immiscible, phase separation is induced on a scale that is directly related to the size of the copolymer chains and results in ordered structures with a domain spacing in the range of 10 to $100 \mathrm{~nm}$. The order/disorder transition temperature and the specific pattern formed by a given BCP are functions of the molecular weight, the strength of interaction between the blocks (represented by the Flory-Huggins interaction parameter, $\chi$ ), and the volumetric composition. In particular, volumetric composition strongly influences microphase morphology. For example, diblock copolymers with segments of comparable volume exhibit a lamellar morphology. Increasing the degree of compositional asymmetry leads to the gyroid, cylindrical, and finally, spherical phases.

When a BCP is dissolved in a selective solvent, i.e., a good solvent for one of the blocks but poor solvent for the other, the BCP self-assembles into micelles. The immiscible block forms the core, while the miscible block forms the corona. The micellar morphology obtained depends on several factors such as length of the blocks, concentration, thermodynamic compatibility between block-solvent and block-block, and temperature (Bang et al. 2009; Wu et al. 2005). When the blocks forming the BCP are amorphous, typical micelle structures include spheres, cylinders, lamellar bilayers 
and vesicles (Hayward and Pochan, 2010; Mai and Eisenberg, 2012). However, it is possible to access to other type of micellar morphologies when the immiscible block is able to crystallize. In this case, crystallization competes with microphase separation in the self-assembly process. (He and Xu, 2012a) When crystal packing forces dominate, a morphological transformation into a novel structure is triggered through a reorganization mechanism called crystallization-driven self-assembly (CDSA). This mechanism normally leads to elongated nanostructures, either with planar cross-sections (nanoribbons or platelet-like structures) or with circular cross-sections (fibers, cylinders). The morphology developed is affected by crystallization temperature and time, solvent quality, core/corona volume ratio and composition (Crassous et al. 2015). Thus, a large variety of morphologies can be prepared by adjusting the crystallization conditions under which semi-crystalline micelles are obtained. Based on this concept, CDSA has emerged as a powerful method for the preparation of well-defined micellar structures with controlled dimensions. A significant number of articles describing the preparation of these elongated nanostructures in solution has been published in recent years. For example, Winnik and Manners found that poly(ferrocenylsilane) (PFS)-based block copolymers self-assemble into cylindrical micelles (Guerin et al. 2014; Shen et al. 2008; Wang et al. 2007) or tape-like nanostructures (Cao et al. 2002) when they are dispersed in alkane solvents. Due to their remaining active ends, these micelles can be elongated, in a controlled manner, through the addition of further block copolymer unimers. It was argued that the process is driven by epitaxial crystallization of the core-forming PFS block on the preformed seed micelles (Boott et al. 2018; Guerin et al. 2018). In other examples, analogous elongated structures with crystallizable organic cores based on poly(ethylene oxide) (PEO) (Mihut et al. 2010), poly(E-caprolactone) (PCL), (He et al. 2012b) and poly(ethylene) (PE) (Yin et al. 2012), were prepared in a controlled way by regulating parameters such as cooling rate and crystallization temperature.

The extension of the original concept of BCP self-assembly in solution to the preparation of nanostructured polymer materials involves the replacement of the selective solvent by reaction precursors. The premise that systems based on blends of diblock or triblock copolymers and reaction precursors must meet to avoid macro phase separation is that at least one of the blocks of the BCP must remain miscible with the matrix during the reaction or it is expelled only on a local scale. Two mechanisms have been identified for the formation of nanostructures depending on the miscibility of the non-affine block of the copolymer with the matrix before and after curing: initial self-assembly and reaction induced microphase separation (RIMPS). With the first mechanism, self-assembled nanophases are present before curing. This occurs when the non-affine block is immiscible in reaction precursors and, consequently, it is phase-separated from the start of the polymerization process. In this case, the role of curing is to "freeze" the existing nanostructure. A variety of $\mathrm{BCP}$ architectures have been used to obtain ordered (or disordered) nanostructures through this strategy (Guo et al. 2003a; Lipic et al. 1998a; Wu et al. 2005). However, in many instances the blocks are miscible in reaction precursors. With the second mechanism, RIMPS, the BCP is initially dissolved in the reactive solvent and the non-affine block phase separates during the polymerization generating the self-assembled nanophases (Meng et al. 2006a).

During the synthesis of nanostructured polymers from a crystallizable BCP, either by initial self-assembly or through the RIMPS mechanism, the nanostructure generated could undergo a morphological transformation to a novel structure through CDSA. This possibility is strictly related to the mobility of the matrix at the time when the crystallization of the immiscible block occurs (Montoya Rojo et al. 2019; Schmarsow et al. 2019). For example, when polymerization occurs above the melting temperature of the immiscible block, crystallization takes place during the final cooling step. Guo et al. (Guo et al. 2003b) analyze this situation. They employed PE- $b$-PEO $\left(M_{\mathrm{n}}=1400 ; 50 \mathrm{wt} \%\right.$ PEO) dispersed in a matrix based on diglycidyl ether of bisphenol A (DGEBA). The cure was performed in steps from $120^{\circ} \mathrm{C}$ to $180{ }^{\circ} \mathrm{C}$ (above the melting temperature of $\mathrm{PE}$, which is around $100^{\circ} \mathrm{C}$ ). For contents up to $30 \mathrm{wt} \% \mathrm{BCP}$, spherical micelles were present during curing, while confined crystallization took place by homogeneous nucleation inside the spherical micelles during the cooling step. Similar results were reported by other authors employing PE- $b$-PEO (Tercjak et al. 2006) or PCL- $b$ PE- $b$-PCL $\left(M_{\mathrm{n}}=31000\right.$ with $35 \mathrm{wt} \%$ PE, where PCL was the miscible block) (Zhang et al. 2013). In all these previous studies, epoxy networks with high glass transition temperature $\left(\mathrm{T}_{\mathrm{g}}\right)$ were selected. As a result, vitrification of the epoxy matrix took place before crystallization during the cooling step. This fixed the nanostructure generated at high temperatures and enabled crystallization to occur in confined domains with rigid boundaries. A different and less studied situation occurs when the $T_{\mathrm{g}}$ of the epoxy network is lower than the crystallization temperature of the immiscible block. (Puig et al. 2016; Schmarsow et al. 2019) In this case, there is a temperature gap between the crystallization and the glass transition of the matrix where CDSA may take place. The size of such temperature gap, which is a measure of the mobility of the matrix, will determine the effect of CDSA on the morphology generated during the cooling step, as will be discussed in this manuscript.

The scenario is different when polymerization occurs below the crystallization temperature of the immiscible block. In this case, two situations could occur. First, if the non-affine block is completely immiscible in reaction monomers, the initial blend will consist of a dispersion of crystalline nanostructures stabilized by the miscible block. In this case, nanostructures are formed through CDSA in the initial blend (Zucchi and Schroeder, 2015; Sinturel et al. 2007, 2009). Sinturel et al. (Sinturel et al. 2007, 2009) employed this mechanism to obtain PE- $b$-PEO platelets with a high aspect ratio ( $6 \mathrm{~nm}$ thick, $500 \mathrm{~nm}$ to $1 \mathrm{~m}$ long) constituted of a crystalline PE core, bordered by PEO domains dispersed in cross-linked unsaturated polyester. The second situation occurs when both blocks of the BCP are miscible in reaction monomers and 
nano-structuration takes place by RIMPS. At the moment, this case remains practically unexplored. Here, the core-forming block can crystallize during polymerization and different nanostructures can be generated depending on the mobility of the matrix when crystallization occurs (Montoya Rojo et al. 2019). The competition between crystallization and polymerization kinetics defines the micellar morphology that develops. This case is particularly interesting since morphologies can be precisely tailored by manipulating curing conditions.

From the discussion above, it is clear that the incorporation of crystallizable polymers as the immiscible core-forming block provides opportunities toward nanostructures with additional structural features. The crystallization energy from the core is responsible for this behavior. The aim of this paper is to review some recent works related to BCP self-assembly in polymer matrices in which the crystallization of the immiscible block plays a critical role in micellar morphology development.

\section{PE-b-PEO self-assembly in an epoxy matrix}

We will begin by showing the results obtained when $10 \mathrm{wt} \%$ of polyethylene-block-poly(ethylene oxide) (PE- $b$-PEO, Mn $=1400$ and $50 \mathrm{wt} \%$ ethylene oxide content) was dispersed in a polymer matrix based on diglycidyl ether of bisphenol A (DGEBA). In this case, the PE block is immiscible with DGEBA and can easily crystallize (Guo et al. 2003b), whereas the PEO block is completely miscible with DGEBA before and after the curing reaction (Meng et al. 2006b). Consequently, BCP nanostructures are formed through the initial self-assembly mechanism.

Firstly, the neat BCP was thermally characterized. Figure 1 shows the DSC cooling scan from the melt and the subsequent heating scan for the pure PE- $b$-PEO. The DSC cooling scan displays a broad distribution of exothermic events with a main peak at $95.9^{\circ} \mathrm{C}$ attributed to the crystallization of the PE blocks, and an exothermic peak at $16.4^{\circ} \mathrm{C}$ attributed to the crystallization of the PEO blocks. This crystallization behavior of the PE blocks is commonly observed in PE-based copolymers obtained via polybutadiene hydrogenation. The DSC subsequent heating scan shows the melting peak of the PEO crystals as an endothermic peak at $30.4^{\circ} \mathrm{C}$, and the melting behavior of the PE crystals as a wide endothermic peak with the minimum at $103.8^{\circ} \mathrm{C}$.

As we previously mentioned, the PE block is immiscible while the PEO block is completely miscible with DGEBA. This marked amphiphilicity induces the formation of micelles with PE core and PEO corona when the BCP is dispersed in DGEBA. The polymerization of DGEBA transfers the obtained micellar dispersion to a cross-linked epoxy matrix. However, the micellar architecture adopted in the thermoset is deeply determined by the conditions in which crystallization occurred. Two different situations can occur. When curing is performed above the melting temperature of the PE block, nanostructures are formed in the molten state during curing, and crystallization occurs during the subsequent cooling step. On the contrary, when curing is carried out at a temperature below the melting transition of the PE block, a dispersion of micelles with already crystallized PE core is present in the initial mixture. These two different scenarios were explored and are summarized below.

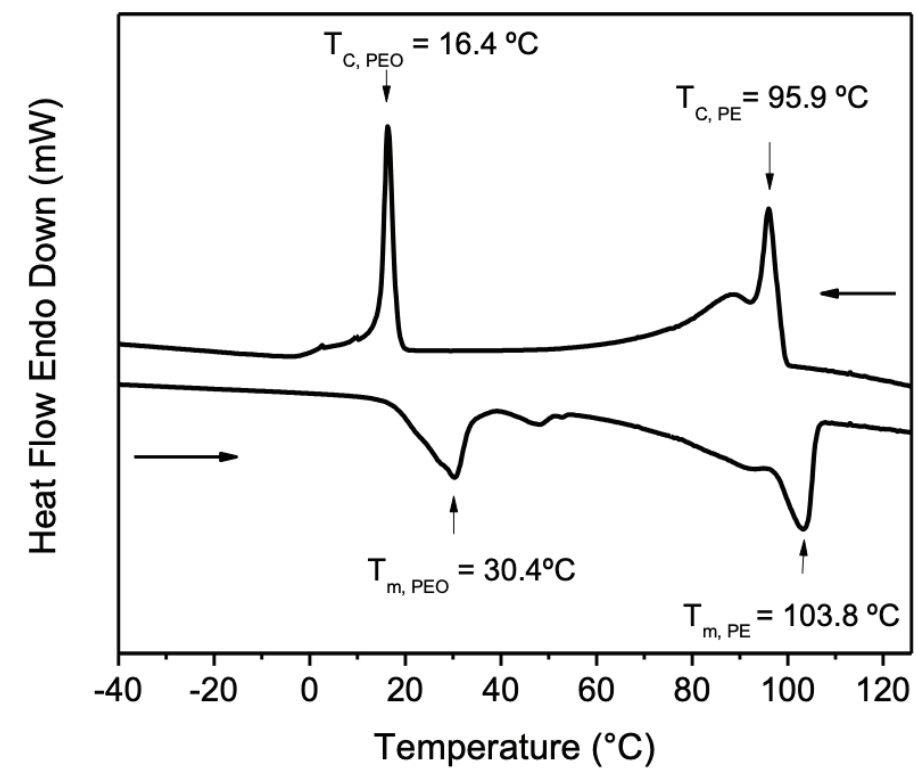

Figure 1. DSC scans at $10{ }^{\circ} \mathrm{C} / \mathrm{min}$ of the cooling (above) and subsequent heating (below) for the pure PE- $b$-PEO block copolymer. 


\subsection{Thermal curing of DGEBA at $120^{\circ} \mathrm{C}$}

To investigate the morphologies obtained when curing was performed above the melting temperature of the PE block, DGEBA was homopolymerized by an anionic mechanism using a tertiary amine benzyldimethylamine (BDMA) as initiator (in a molar ratio with respect to epoxy groups equal to 0.06 ). The reaction was carried out at $120^{\circ} \mathrm{C}$. At this temperature, 2 hours of reaction were sufficient to achieve complete conversion. Figure 2(a) shows a TEM image of $\mathrm{RuO}_{4}$-stained sample of the fully cured blend containing $10 \mathrm{wt} \%$ BCP. The presence of micelles with ellipsoidal contours of approximately $20 \mathrm{~nm}$ in length is observed in the TEM image. PEO blocks are preferentially stained by $\mathrm{RuO}_{4}$, compared to the PE cores and the epoxy matrix (Trent et al. 1983), therefore PEO-rich regions (contour of the micelles) appear darker in the TEM image.
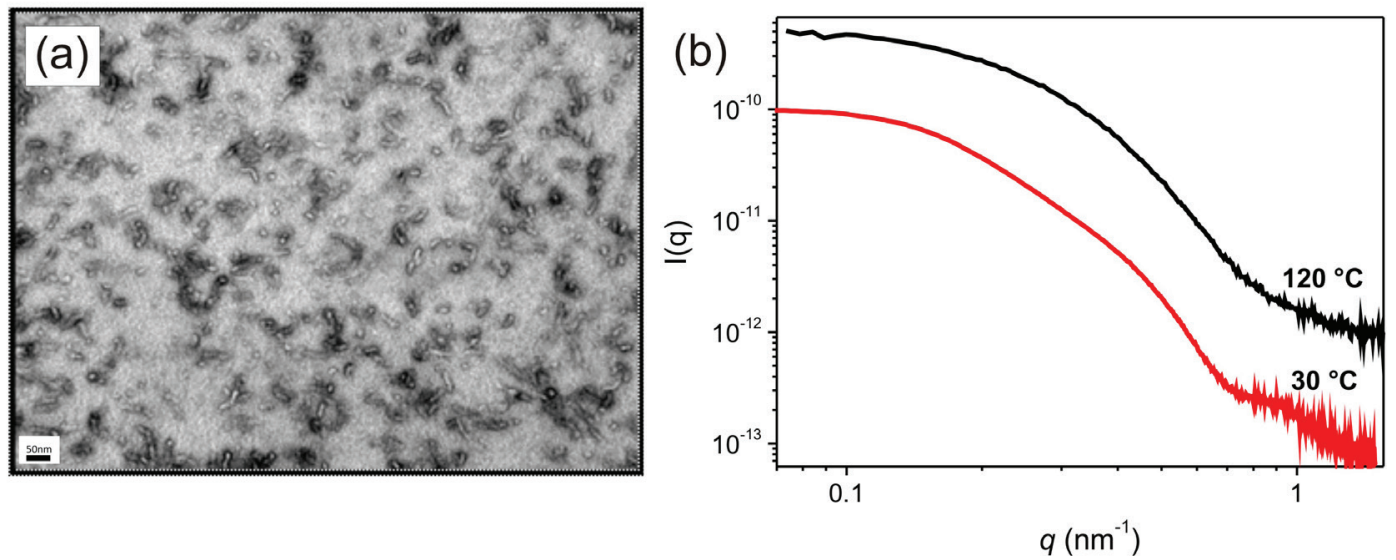

Figure 2. (a) TEM image of the fully cured blend containing $10 \mathrm{wt} \% \mathrm{PE}-b-\mathrm{PEO}$, scale bar $50 \mathrm{~nm}$. (b) SAXS data obtained after polymerization at $120^{\circ} \mathrm{C}$ and subsequent cooling to $30^{\circ} \mathrm{C}$ of $10 \mathrm{wt} \% \mathrm{PE}-b$-PEO/epoxy blend. [Puig, J.; Zucchi, I. A.; Ceolín, M.; Schroeder, W. F.; Williams, R. J. J. RSC Adv 2016, 6 (41), 34903-34912] Reproduced by permission of The Royal Society of Chemistry.

The SAXS curve recorded at $120^{\circ} \mathrm{C}$, after the reaction and before the cooling step, is displayed on Figure 2(b) (top curve). Curves were fitted using the SASfit software package, in the region from 0.08 to $0.5 \mathrm{~nm}^{-1}$ (where the contribution of the neat matrix showed a negligible intensity). The analysis of SAXS data required a population of short cylinders with an average diameter of $8 \mathrm{~nm}$ and average length of $23 \mathrm{~nm}$. These cylinders, which are one of the typical nanostructures generated when polymerization is carried out above the melting temperature of the immiscible block, were generated during thermal curing at $120^{\circ} \mathrm{C}$.

Then, it was investigated whether the crystallization-driven self-assembly of PE blocks produced any change in the morphology when cooling the cured material from $120^{\circ} \mathrm{C}$ to room temperature. The bottom curve in Figure 2(b) displays the SAXS curve of the fully cured blend after cooling to $30^{\circ} \mathrm{C}$. The fitting required to assume the presence of a polydisperse distribution of planar objects (disk-like micelles) with an average diameter $d_{\text {disks }}=23.1 \mathrm{~nm}$ and thickness $h$ $=6.7 \mathrm{~nm}$. The thickness is close to the theoretical value of fully extended PE chains (Parikh et al. 1999). IR spectroscopy confirmed the all-trans conformation of PE chains. Therefore, crystals were formed by interdigitated PE chains, with PEO blocks tethered at both planar interfaces in an alternating way. SAXS analysis together with TEM image (Figure 2(a)), allowed to conclude that planar nanostructures can be described as disk-like micelles with ellipsoidal contours that preserve the original size of the cylinders. Cooling led to a change in shape of the individual micelles, from nano-rods to disk-like objects, promoted by the crystallization of PE chains.

As it was mentioned before, during the crystallization of the immiscible block the matrix should have enough molecular mobility to allow the CDSA mechanism to operate. A measure of such molecular mobility is given by the temperature gap between the crystallization of PE blocks and the glass transition of the matrix $\left({ }_{T c, P E}-T_{g, \text { matrix }}\right)$. The DSC heating and cooling scans of the fully cured blend containing $10 \mathrm{wt} \%$ PE- $b$-PEO allow measuring such temperature gap, see Figure 3. The heating scan showed the $T_{\mathrm{g}}$ of the plasticized epoxy at $58^{\circ} \mathrm{C}$ (onset value), which was significantly lower than the value for neat epoxy $\left(81^{\circ} \mathrm{C}\right.$, not shown) due to PEO plasticization. PE crystals melted at $98^{\circ} \mathrm{C}$ (minimum of the endothermic peak). The cooling scan showed a crystallization peak at $65^{\circ} \mathrm{C}$. Therefore, this blend exhibited a very narrow temperature gap, from about $70{ }^{\circ} \mathrm{C}$ (onset of crystallization) to $58^{\circ} \mathrm{C}$, to produce CDSA. This temperature gap only allowed flattening of the domains, but not elongation of the nanostructure. 


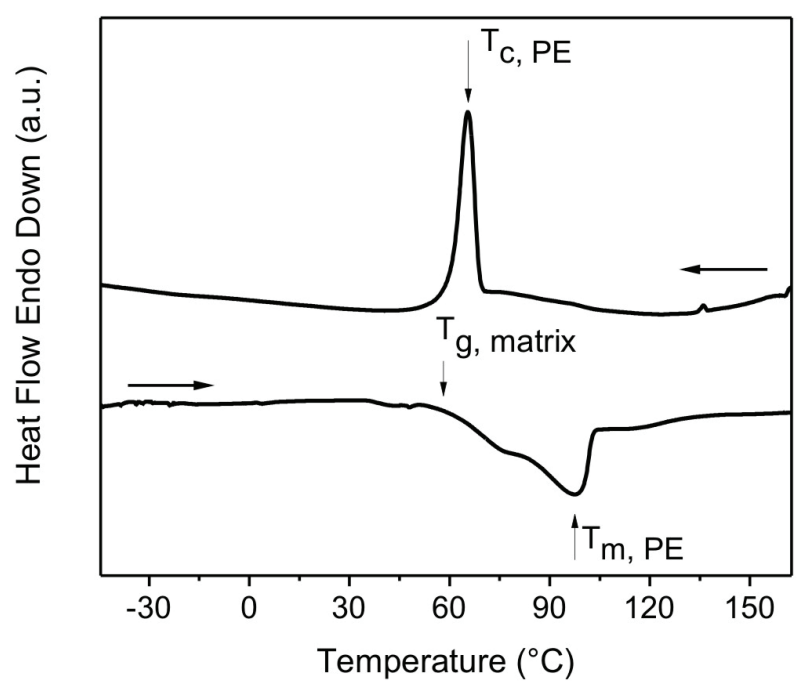

Figure 3. DSC thermogram in heating and cooling scans at $10{ }^{\circ} \mathrm{C} / \mathrm{min}$ for the cured blend with $10 \mathrm{wt} \% \mathrm{PE}-b$-PEO. [Puig, J.; Zucchi, I. A.; Ceolín, M.; Schroeder, W. F.; Williams, R. J. J. RSC Adv 2016, 6 (41), 34903-34912] Reproduced by permission of The Royal Society of Chemistry.

\subsection{Photopolymerization of DGEBA at room temperature}

To investigate the morphologies obtained when curing temperature is below the melting transition of the PE block, epoxy monomers were photocured at room temperature by photoinitiated cationic ring-opening polymerization. The resin was activated for visible light polymerization by adding a three-component photoinitiating system based on $p$-(octyloxyphenyl) phenyliodonium hexafluoroantimonate $\left(\mathrm{Ph}_{2} \mathrm{ISbF}_{6}, 2\right.$ wt \%), camphorquinone (CQ, 1 wt \%) and ethyl-4-dimethyl aminobenzoate (EDMAB, $1 \mathrm{wt} \%$ ). Under these conditions, DGEBA undergoes very slow polymerization at room temperature. Between three and four days of irradiation were required to reach a high enough conversion level.
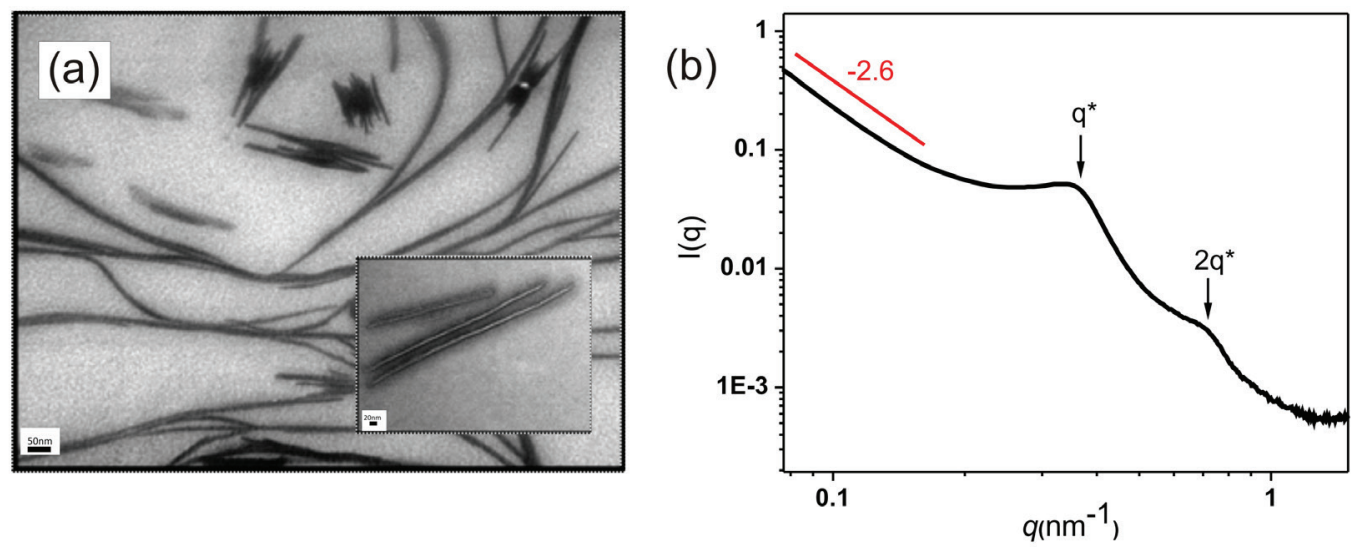

Figure 4. (a) TEM images of $\mathrm{RuO}_{4}$-stained section of the photocured blend with $10 \mathrm{wt} \% \mathrm{PE}-b$-PEO, scale bar $50 \mathrm{~nm}$. Inset: TEM image of the same sample at a higher magnification, scale bar $20 \mathrm{~nm}$. (b) SAXS profile of the photocured blend with 10 wt \% PE- $b$-PEO.

Figure 4(a) shows TEM images of $\mathrm{RuO}_{4}$-stained sections of the photocured sample containing $10 \mathrm{w} \% \mathrm{BCP}$. A dispersion of nanoribbons with semi-crystalline core dispersed in the epoxy matrix was obtained. As explained above, PEO is preferentially stained by $\mathrm{RuO}_{4}$ compared to PE and DGEBA; therefore, PEO-rich regions look darker in the TEM image (Trent et al. 1983). Furthermore, the staining technique allows clearly distinguishing the location of the blocks in the structure. The TEM image at higher magnification (inset in Fig. 4(a)) shows micelles viewed edge-on exhibiting the PEO corona as darker lines surrounding the PE core (lighter line). An interesting feature of these ribbon-like micelles was their tendency to aggregate, resulting in the formation of face-to-face stacking of micelles as shown in Figure 4(a). This 
association effect may be driven by the attractive van der Waals interaction between different nanoribbons. SAXS measurement was carried out to extract complementary structural information, Figure 4(b). The first interesting feature is the low-q slope, which was equal to -2.6 , evidencing the presence of elongated planar nanostructures. The pattern presented a main peak located at a value of the scattering vector $q^{*}=0.38 \mathrm{~nm}^{-1}$, corresponding to an average spacing of $16.5 \mathrm{~nm}$. This main peak was accompanied by a well-defined higher order reflection located at $q_{i} / q^{*}=2$, where $q^{*}$ is the primary peak position. Such a sequence is characteristic of a lamellar arrangement. The results from the combination of TEM and SAXS allow to conclude that the ribbon-like micelles adopted face-to-face packing into lamellar arrangements with an average period of $16.5 \mathrm{~nm}$.

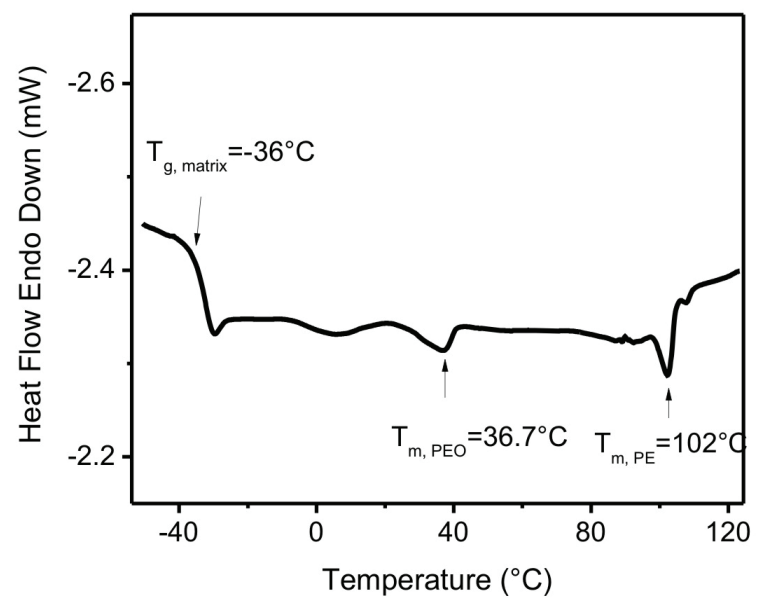

Figure 5. DSC thermogram in heating scan at $10^{\circ} \mathrm{C} / \mathrm{min}$ of the initial $10 \mathrm{wt} \% \mathrm{PE}-b$-PEO/DGEBA blend.

In the mixture that was photopolymerized at room temperature, crystallization of PE blocks took place in the reactive monomers before polymerization, while the subsequent curing preserved the crystalline structure of the generated micelles. In this case, the temperature gap for crystallization-driven self-assembly $\left(T_{\mathrm{c}, \mathrm{PE}}-T_{\mathrm{g} \text {,matrix }}\right)$ was $138^{\circ} \mathrm{C}$, which corresponds to the difference between the crystallization temperature of PE blocks $\left(102^{\circ} \mathrm{C}\right)$ and the glass transition of the monomers $\left(-36^{\circ} \mathrm{C}\right)$, as shown in Figure 5. For this large temperature gap, long crystalline nanoribbons of several microns in length were obtained in the initial mixture. These nanoribbons were subsequently frozen by the photocuring reaction of the matrix.

\subsection{Combined Thermal- and Photo-Curing}

The results presented in these two previous sections highlight the crucial role of matrix mobility during immiscible PE blocks crystallization in the elongation process of the planar nanostructures, where the temperature gap $\left(T_{\mathrm{c}, \mathrm{PE}}-T_{\mathrm{g}, \text { matrix }}\right)$ provides a measure of such mobility.

On the basis of this finding, it was proposed to obtain a dispersion of nanoribbons with precisely controlled length by regulating the temperature gap $\left(T_{\mathrm{c}, \mathrm{PE}}-T_{\mathrm{g} \text {,matrix }}\right)$ during PE blocks crystallization. For this, a dual thermal- and photo-curing system was adopted, which allowed initiating the epoxy polymerization at $120{ }^{\circ} \mathrm{C}$ until a certain degree of conversion, stopping the reaction by cooling to induce micellar elongation and crystallization, and then continuing the polymerization process at room temperature by visible-light irradiation. In this way, PE blocks crystallization took place in a matrix whose mobility was controlled by the degree of conversion reached at $120^{\circ} \mathrm{C}$. Figure 6 shows the temperature gap between the main peak crystallization temperature of PE blocks $\left(T_{\mathrm{c}, \mathrm{PE}}\right)$ and glass transition temperature of the epoxy matrix $\left(T_{\text {g,matrix }}\right)$ measured from DSC scans, as a function of the conversion degree reached during the thermal polymerization step (xt) for a sample with $10 \mathrm{wt} \%$ BCP. As observed in Figure 6, matrix mobility can be effectively manipulated by regulating the degree of conversion reached at $120^{\circ} \mathrm{C}$. 


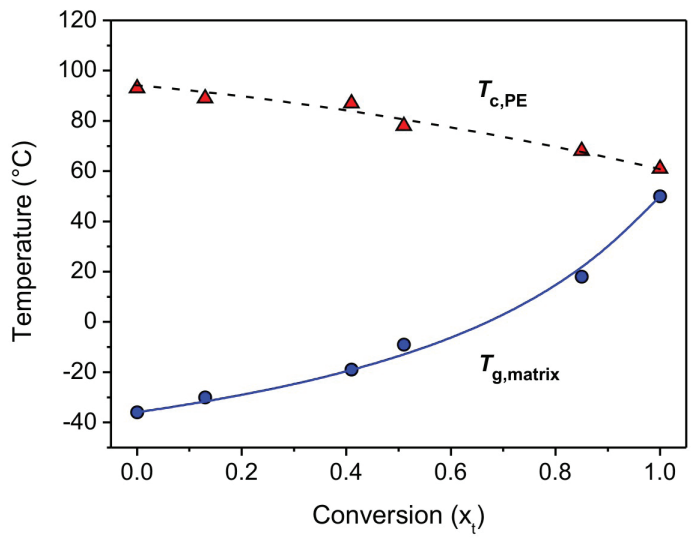

Figure 6. Main peak crystallization temperature of PE blocks ( $T_{\mathrm{c}, \mathrm{PE}}$, red triangles) and glass transition temperature of the epoxy matrix ( $T_{\mathrm{g} \text {,matrix }}$, blue circles) obtained from DSC scans versus conversion. The blue solid line represents the evolution of $T_{\mathrm{g} \text {,matrix }}$ predicted by equation $\left(T_{\mathrm{g}}-T_{\mathrm{g} 0}\right) /\left(T_{\mathrm{g} \infty}-T_{\mathrm{g} 0}\right)=\lambda \mathrm{x} /[1-(1-\lambda) x]$, with $\lambda=0.348, T_{\mathrm{g} 0}=-36^{\circ} \mathrm{C}$ and $T_{\mathrm{g} \infty}$ $=50^{\circ} \mathrm{C}$. The dashed line was drawn to guide the eye. [Schmarsow, R. N.; Ceolín, M.; Zucchi, I. A.; Schroeder, W. F. Soft Matter 2019, 15, 4751-4760] Reproduced by permission of The Royal Society of Chemistry.

Figure 7 shows TEM images of the materials obtained with different conversion degrees reached during the thermal polymerization step. For comparison purposes, the images are shown at the same magnification. The sectioned specimens were stained with $\mathrm{RuO}_{4}$ prior to TEM observation. As it can be seen, average micelle length decreased progressively as $\mathrm{x}_{\mathrm{t}}$ increased. These results confirmed that average micelle length decreased as the difference between $T_{\mathrm{c}, \mathrm{PE}}$ and $T_{\mathrm{g} \text {,matrix }}$ decreased, which was a consequence of medium mobility loss during the crystallization process. The dimensions obtained by TEM analysis were consistent with the visual appearance of the samples. Figure 7 (insets) shows photographs of the prepared materials. As it can be observed, the fully photocured sample $\left(x_{t}=0\right)$ was opaque due to the presence of ribbon-like micelles with lengths at the micron scale. With increasing $x_{t}$, there was a gradual decrease in opacity, indicating that micelle size progressively decreased below the wavelength of visible light. For $x_{t}=0.85$ and 1 , the samples were fully transparent, as expected for a dispersion of nanoscale objects.
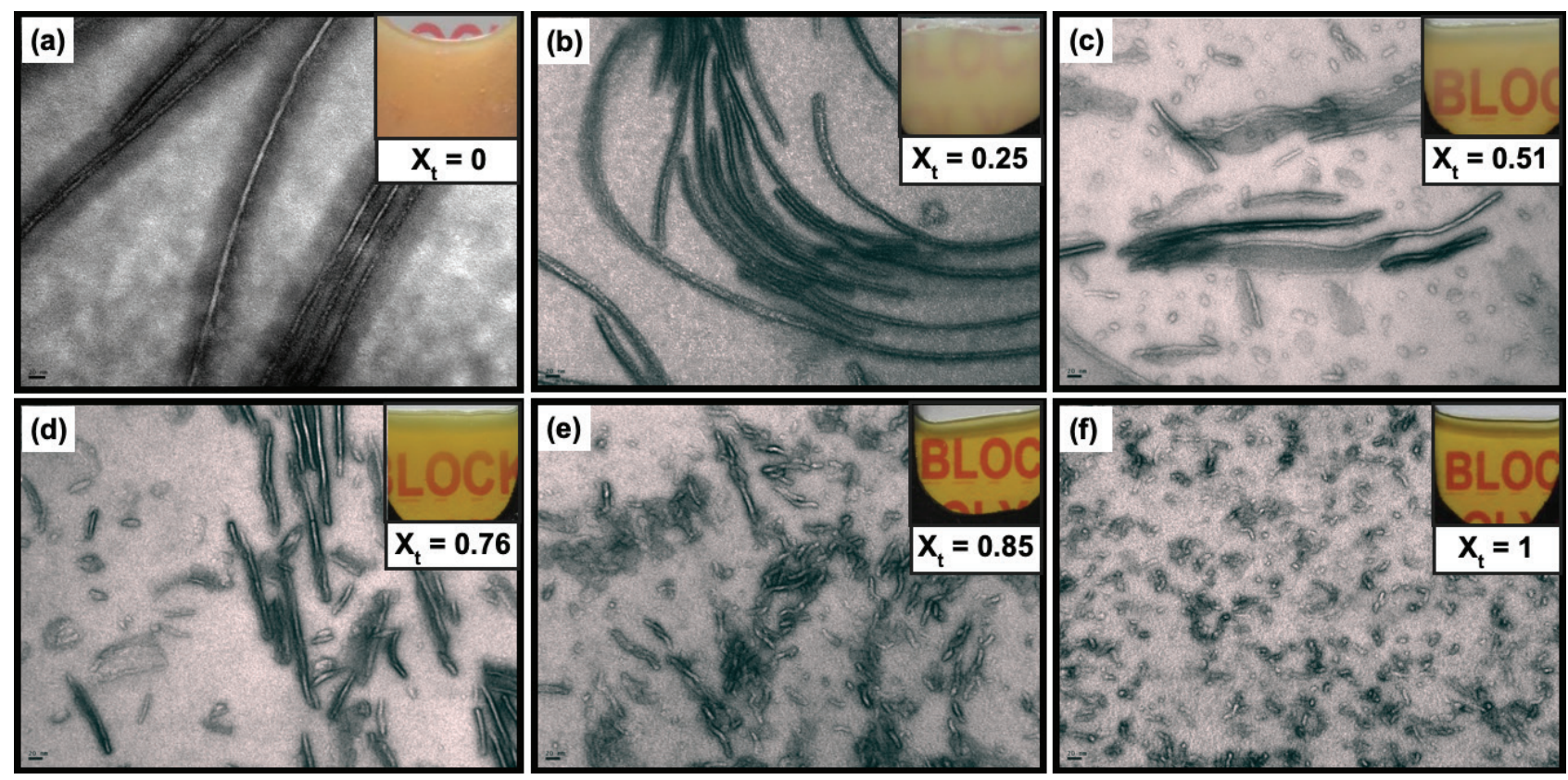

Figure 7. TEM images of materials with $10 \mathrm{wt} \%$ PE- $b$-PEO obtained with different conversion degrees achieved during the thermal polymerization step $\left(\mathrm{x}_{\mathrm{t}}\right)$. Sectioned specimens were stained with $\mathrm{RuO}_{4}$ prior to the TEM observations. In all the images, the scale bar represents $20 \mathrm{~nm}$. The insets show photographs of the corresponding materials. (a) $x_{t}=0$; (b) $x_{t}$ $=0.25 ;(\mathrm{c}) \mathrm{x}_{\mathrm{t}}=0.51 ;$ (d) $\mathrm{x}_{\mathrm{t}}=0.76 ;$ (e) $\mathrm{x}_{\mathrm{t}}=0.85$; and (f) $\mathrm{x}_{\mathrm{t}}=1$. [Schmarsow, R. N.; Ceolín, M.; Zucchi, I. A.; Schroeder, W. F. Soft Matter 2019, 15, 4751-4760] Reproduced by permission of The Royal Society of Chemistry. 
These results showed that ribbon-like micelles with precisely controlled length can be obtained through a crystallization-driven self-assembly mechanism. Micellar length can be precisely tailored by regulating $T_{\mathrm{c}, \mathrm{PE}}-T_{\mathrm{g}, \text { matrix }}$. Figure 8 summarizes the preparation conditions that lead to the length-controlled planar nanostructures.

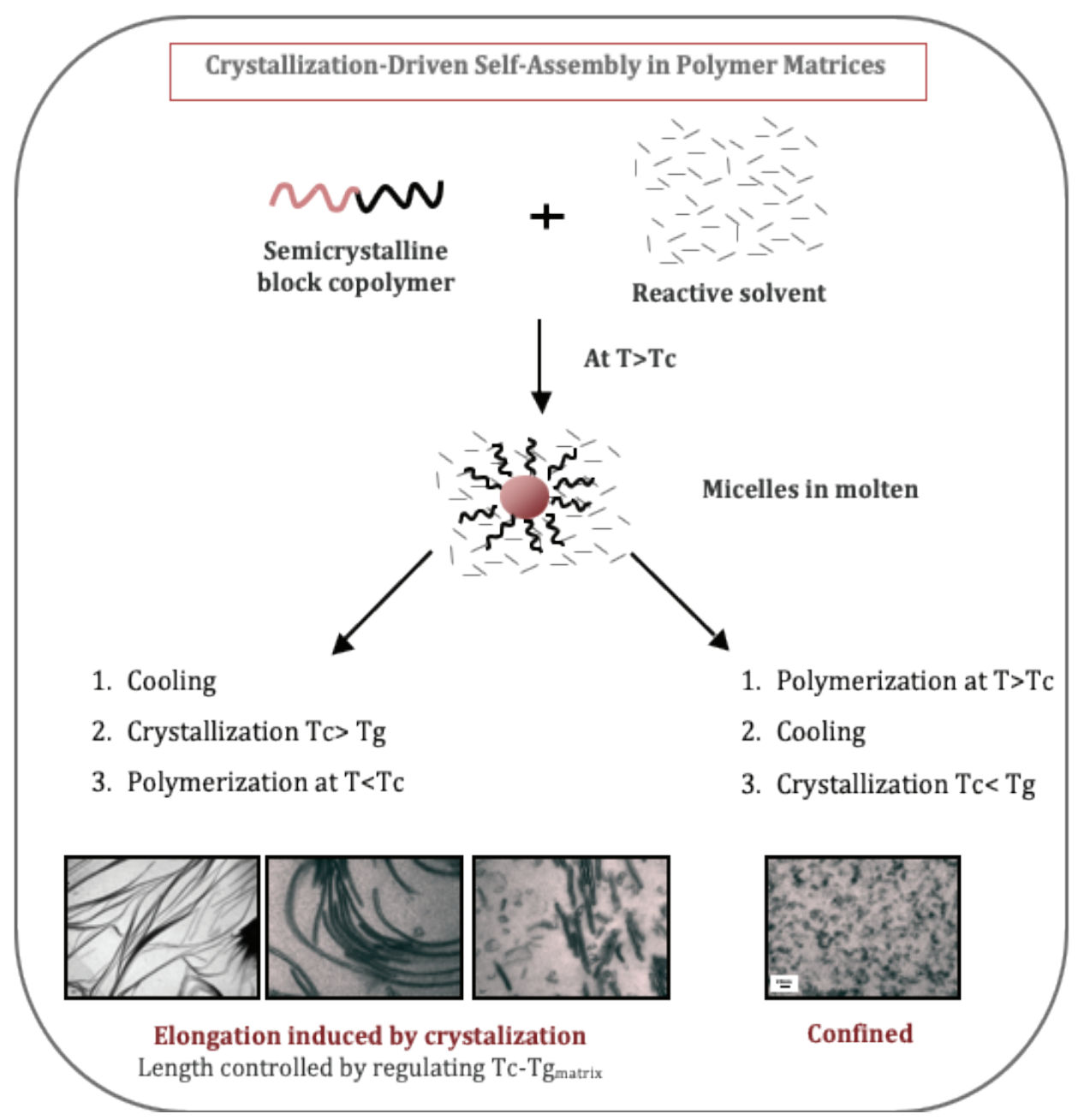

Figure 8. Schematic representation of the different pathways followed for the preparation of the elongated micelles.

\section{PS-b-PCL self-assembly in a polystyrene matrix}

We will continue by analyzing a completely different scenario. In this case, with the goal of reducing the crystallization ability of the immiscible block, PCL was used as core-forming block in an amphiphilic combination with PS. The BCP employed was Poly(styrene-block- $\varepsilon$-caprolactone) (PS- $b$-PCL) with $38 \mathrm{wt} \%$ PCL and $\mathrm{Mn}=47,000 \mathrm{~g} / \mathrm{mol}(\mathrm{Mw} / \mathrm{Mn}=$ 1.02). Consequently, the reactive solvent must be non-affine with PCL to cause its de-mixing and therefore the self-assembly. Taking this into account, the selected reactive solvent was styrene ( $\mathrm{St}$ ), which was a good solvent for both blocks before reaction, but became a poor solvent for the PCL block during the polymerization. This condition shows that the system will be nanostructured by the RIMPS mechanism. Samples were prepared at two different temperatures of polymerization, 90 and $20^{\circ} \mathrm{C}$, i.e. above and below the melting temperature of the PCL block. 


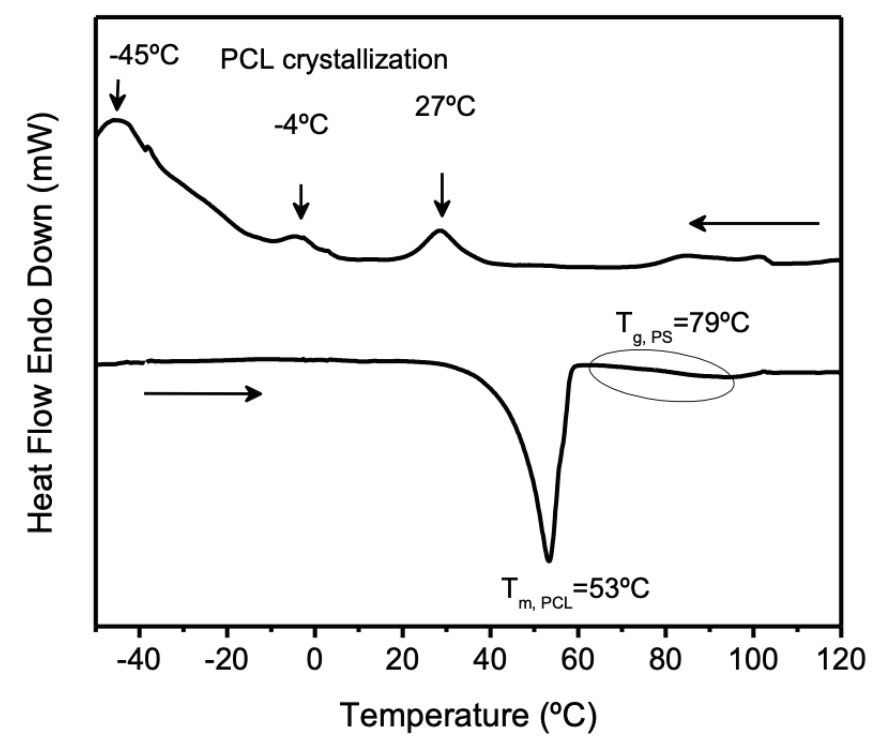

Figure 9. DSC scans at $10^{\circ} \mathrm{C} / \mathrm{min}$ of the cooling phase (top) and subsequent heating (down) for the pure PS- $b$-PCL block copolymer. Reprinted from Eur. Polym. J., 112, Montoya Rojo, Ú. M.; Riccardi, C. C.; Ninago, M. D.; Ciolino, A. E.; Villar, M. A.; Ceolín, M.; Zucchi, I. A.; Schroeder, W. F., Photopolymerization-Assisted Self-Assembly as a Strategy to Obtain a Dispersion of Very High Aspect Ratio Nanostructures in a Polystyrene Matrix, 704-713, Copyright (2019), with permission from Elsevier.

As a reference, DSC curves for the neat PS- $b$-PCL were first analyzed, see Figure 9. According to its volumetric composition, this block copolymer self-assembles into cylinders of PCL dispersed in a matrix of PS. In the cooling DSC scan shown in Figure 9, several crystallization exotherms were observed, with distinguishable peaks at $27,-4$ and $-45^{\circ} \mathrm{C}$. This behavior was ascribed to a fractionated crystallization phenomenon produced by the confinement of PCL into isolated cylindrical microdomains and by the existence of different nucleation events within such microdomains (Michell and Müller, 2016). Typically, this phenomenon occurs when the number of microdomains is larger than the number of heterogeneities available to produce nucleation. Therefore, the exothermic peak at $27{ }^{\circ} \mathrm{C}$ was assigned to the crystallization of microdomains containing highly active heterogeneities (those commonly present in bulk PCL), while the exothermic peak at $-4{ }^{\circ} \mathrm{C}$ was related to the crystallization of microdomains with less active heterogeneities, which required a higher degree of supercooling. The peak at $-45^{\circ} \mathrm{C}$ was ascribed to the heterogeneity-free microdomains that crystallized by a homogeneous nucleation process. Note that the homogeneous nucleation should occur at the maximum degree of supercooling just before vitrification ( $T_{\mathrm{g}}$ of PCL is approximately $-60^{\circ} \mathrm{C}$ ). (Tiptipakorn et al. 2015) DSC subsequent heating scan (Figure 9) presented an endothermic peak at $54^{\circ} \mathrm{C}$ due to the melting of PCL crystals, followed by the $T_{\mathrm{g}}$ of PS blocks at $79{ }^{\circ} \mathrm{C}$ (onset value).

\subsection{Thermal Polymerization at $90^{\circ} \mathrm{C}$}

Firstly, the self-assembly behavior of the PS-b-PCL during St polymerization at $90{ }^{\circ} \mathrm{C}$ was examined. The polymerization was carried on employing benzoyl peroxide ( $2 \mathrm{wt} \%$ ) as thermal initiator. Under the selected polymerization conditions, almost full conversion of vinyl double bonds was achieved after 70 min reaction.

Previous studies performed using a PCL homopolymer with a molar mass similar to that of the PCL block in the BCP, confirmed that PCL was initially miscible in St monomer and phase separated during polymerization at a conversion degree between 0.1 and 0.2. Consequently, in the blend containing $10 \mathrm{wt} \%$ PS- $b$-PCL, although both blocks (PCL and PS) were initially miscible with the St monomer, the PCL block will phase separate during St polymerization, resulting in self-assembled structures driven by the RIMPS mechanism. Figure 10(a) shows a $\mathrm{RuO}_{4}$-stained TEM micrograph of the sample with $10 \mathrm{wt} \%$ PS- $b$-PCL polymerized at $90^{\circ} \mathrm{C}$. As it can be seen, the micrograph reveals the presence of a population of nanorods dispersed in the PS matrix, with the PS blocks forming the corona of the micelles and the PCL blocks forming the core. The contour length of these micelles ranged from 30 to $220 \mathrm{~nm}$. 

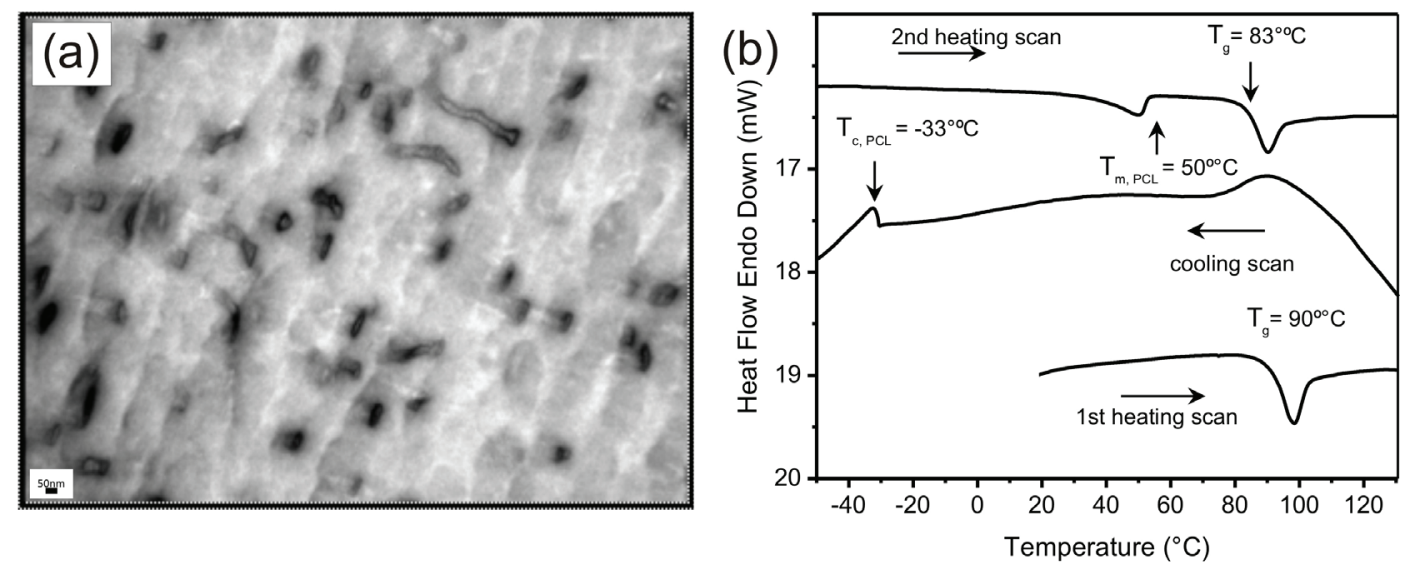

Figure 10. (a) TEM image of the blend with $10 \mathrm{wt} \% \mathrm{PS}-b$-PCL cured at $90^{\circ} \mathrm{C}$. The specimen was stained with $\mathrm{RuO}_{4}$ prior to the TEM observation. Scale bar $50 \mathrm{~nm}$. (b) DSC heating and cooling scans of the fully cured blend with $10 \mathrm{wt}$ \% PS-b-PCL. Reprinted from Eur. Polym. J., 112, Montoya Rojo, Ú. M.; Riccardi, C. C.; Ninago, M. D.; Ciolino, A. E.; Villar, M. A.; Ceolín, M.; Zucchi, I. A.; Schroeder, W. F., Photopolymerization-Assisted Self-Assembly as a Strategy to Obtain a Dispersion of Very High Aspect Ratio Nanostructures in a Polystyrene Matrix, 704-713, Copyright (2019), with permission from Elsevier.

Figure 10(b) shows DSC runs of the cured sample. The first heating scan (at $\left.10^{\circ} \mathrm{C} / \mathrm{min}\right)$, performed from room temperature to $130{ }^{\circ} \mathrm{C}$, exhibited only a thermal transition associated to the glass transition of the matrix $\left(\mathrm{T}_{\mathrm{g}}=90^{\circ} \mathrm{C}\right)$. The absence of a melting peak corresponding to phase-separated PCL blocks indicated that the crystallization of these blocks did not occur during cooling of the sample to room temperature after polymerization. In fact, DSC tests were periodically performed in order to investigate whether confined crystallization of PCL cores took place during sample annealing at room temperature, but crystallization was not detected after four months of annealing. However, PCL crystallization was detected when the sample was subjected to large undercoolings during the subsequent cooling scan. On the basis of these results, it can be understood that PCL blocks were purified by recrystallization during the RIMPS process, resulting in heterogeneity-free microdomains that crystallized by a homogeneous nucleation phenomenon. Consequently, the crystallization peak at $27^{\circ} \mathrm{C}$ was suppressed and crystallization occurred at $-33^{\circ} \mathrm{C}$. Rod-like micelles with an amorphous PCL core dispersed in a PS matrix were the result of thermal curing at $90^{\circ} \mathrm{C}$. A different situation occurred when the reaction was performed at a temperature below the melting temperature of the core-forming block. In this case, the immiscible block was able to crystallize during the polymerization reaction and crystal packing forces played a dominant role in determining the morphology of the nanostructures that were formed. This case is analyzed in the following section.

\subsection{Photopolymerization at Room Temperature}

When polymerization is carried out below melting transition of the demixing block, it can crystallize during the reaction. This is a particularly interesting case because the morphology could be manipulated by controlling the curing conditions.

A blend containing $10 \mathrm{wt} \%$ PS- $b$-PCL was photocured at room temperature (i.e. below the melting temperature of the PCL block, which is $53^{\circ} \mathrm{C}$; see Figure 9) by free radical polymerization. The monomer was activated for visible light polymerization by the addition of CQ ( $2 \mathrm{wt} \%$ ) and EDMAB ( $2 \mathrm{wt} \%)$. A vitrification conversion of 0.88 was reached after four days of irradiation. As in the case of thermal curing, PCL was initially miscible with St monomer and phase-separated during photopolymerization at room temperature at a conversion degree between 0.1 and 0.2 . Figure 11(a) shows a TEM image of the sample with $10 \mathrm{wt} \%$ PS- $b$-PCL photopolymerized at room temperature. Elongated micelles with lengths ranging from $300 \mathrm{~nm}$ to more than $2 \mu \mathrm{m}$, coexisting with much shorter micelles of about $60 \mathrm{~nm}$ in length, were observed. It was also noted that elongated micelles tended to be parallel to each other, resulting in face-to-face stacking into lamellar arrangements. This feature evidenced the planar nature of these structures, which can be described as ribbon-like micelles. Further information on these nanostructures was obtained by analyzing the SAXS profile recorded on the cured material (Figure 11(b)). The magnitude of the slope in the low- $q$ region (Guinier regime) was equal to -2.2., which indicated the presence of planar nano-objects (Nakano et al. 1999). The scattering profile displayed a main maximum of spatial correlation at $q^{*}=0.14 \mathrm{~nm}^{-1}$, and a secondary maximum located at $2 q^{*}$. Such a sequence corresponds to a lamellar arrangement featuring an average distance between nano-objects of $44.9 \mathrm{~nm}\left(2 \pi / q^{*}\right)$, which was in accordance with the lamellar period estimated from the TEM image. 

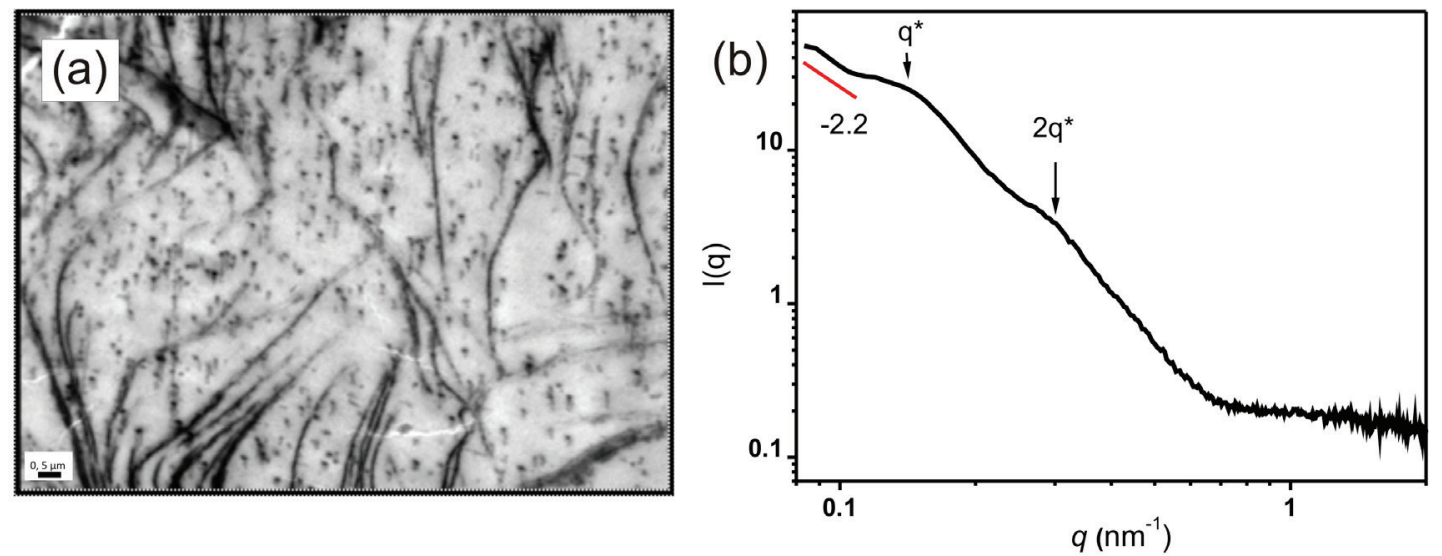

Figure 11. (a) TEM image of the blend with $10 \mathrm{wt} \%$ PS- $b$-PCL photocured at room temperature. The specimen was stained with $\mathrm{RuO}_{4}$ prior to TEM observation. Scale bar $0.5 \mu \mathrm{m}$. (b) SAXS profile of the blend with 10 wt $\%$ PS- $b$-PCL photocured at room temperature. Reprinted from Eur. Polym. J., 112, Montoya Rojo, Ú. M.; Riccardi, C. C.; Ninago, M. D.; Ciolino, A. E.; Villar, M. A.; Ceolín, M.; Zucchi, I. A.; Schroeder, W. F., Photopolymerization-Assisted Self-Assembly as a Strategy to Obtain a Dispersion of Very High Aspect Ratio Nanostructures in a Polystyrene Matrix, 704-713, Copyright (2019), with permission from Elsevier.

To investigate the nanostructuration process of PS- $b$-PCL during the photopolymerization of St, in-situ SAXS and XRD experiments were performed. The results showed that, during the first 41 hours of irradiation, no events occurred except for the demixing of PCL blocks, which took place at 15 minutes of reaction. PCL cores crystallization occurred after 41 hours of irradiation. This process was evidenced as a marked raise in the intensity of SAXS profiles caused by the increase in phase contrast when one of phases crystallizes. It should be clarified that the $\mathrm{T}_{\mathrm{g}}$ of the matrix corresponding to 41 hours of irradiation was approximately $0{ }^{\circ} \mathrm{C}$ (as determined by DSC, data not shown), which is below the crystallization temperature of PCL $\left(27^{\circ} \mathrm{C}\right)$. Under these conditions, the temperature gap $\left(T_{\mathrm{c}, \mathrm{PCL}}-T_{\mathrm{g} \text {,matrix }}\right)$ at the beginning of the crystallization process was $27^{\circ} \mathrm{C}$. This temperature window was sufficient for the CDSA mechanism to cause micelle elongation. This micellar elongation process took place during the time elapsed between matrix crystallization and vitrification. Note that if the polymerization kinetics had not been slow enough, matrix vitrification could have occurred before micellar crystallization, resulting in amorphous rod-like micelles frozen in the PS matrix, similar to the sample polymerized at 90 ${ }^{\circ} \mathrm{C}$. Consequently, it was stated that under slow photopolymerization at room temperature, a blend consisting of $10 \mathrm{wt} \%$ PS- $b$-PCL dissolved in St monomer led to a population of long crystalline nanoribbons dispersed in a PS matrix.

\section{PEB-b-PEO self-assembly in an epoxy matrix}

Lastly, to explore the effect of suppressing immiscible block crystallization, poly(ethylene-co-butene)-block-poly(ethylene oxide) (PEB- $b$-PEO, $M_{\mathrm{n}}=2700, \mathrm{PDI}=1.09,45 \mathrm{wt} \%$ PEB) was chosen as block copolymer and DGEBA as the reactive monomer. In this case, PEB block is completely immiscible with DGEBA, while PEO is miscible before and after the curing reaction. Consequently, nanostructures were obtained from the initial blend through the self-assembly mechanism. Samples were prepared at two different temperatures of polymerization, $80^{\circ} \mathrm{C}$ and room temperature $\left(20^{\circ} \mathrm{C}\right)$. 


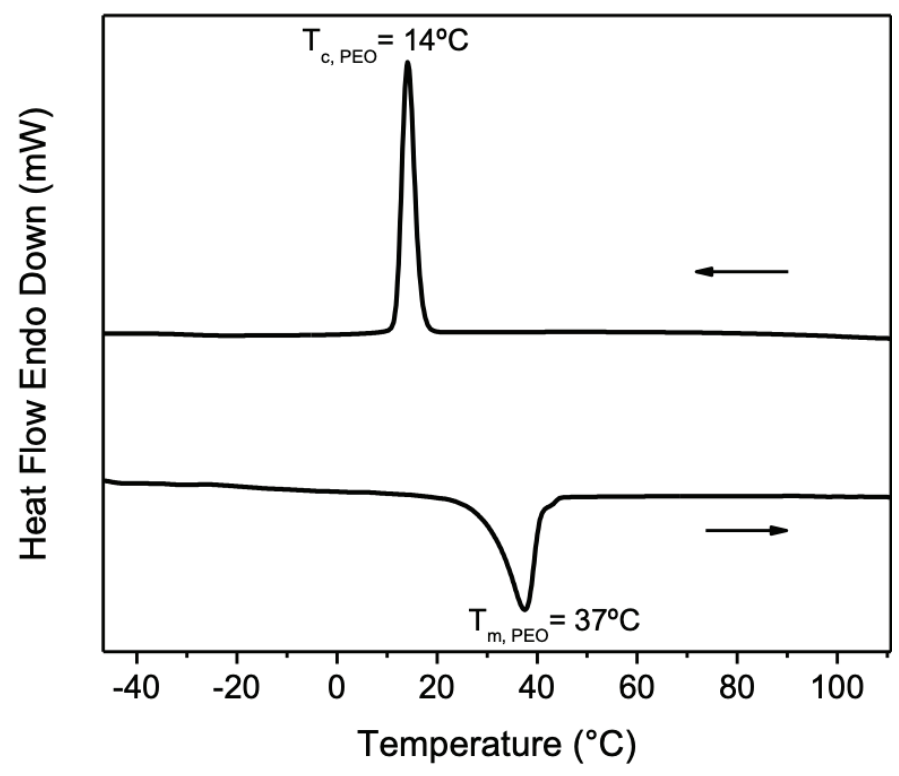

Figure 12. DSC scans at $10{ }^{\circ} \mathrm{C} / \mathrm{min}$ of the cooling (above) and subsequent heating (below) for the pure PEB- $b$-PEO block copolymer.

The DSC analysis of the neat PEB- $b$-PEO allowed to corroborate the absence of crystallinity of the immiscible block (PEB). As it can be seen in Figure 12, only one thermal transition was observed during cooling and heating scans, assigned to crystallization and melting of PEO crystals, respectively. The location of such transitions was in agreement with the molecular weight of PEO blocks (Leonardi et al. 2015). The DSC analysis confirmed that the PEB block was amorphous, and its glass transition temperature was located below the temperature range explored.

\subsection{Curing at $80^{\circ} \mathrm{C}$}

First, the case in which a $10 \mathrm{wt} \%$ of PEB- $b$-PEO/DGEBA blend was thermally cured at $80^{\circ} \mathrm{C}$ is discussed. Here, BDMA was used as thermal initiator for epoxy homopolymerization. Under these conditions, the reaction was completed after 3 hours (Puig et al. 2017). Figure 13(a) shows a TEM image of the material obtained after curing. A dispersion of spherical micelles partially arranged in micellar columns and some cylindrical micelles were observed. In order to get information about the evolution of such nanostructures along polymerization, in situ SAXS spectra were acquired during polymerization at $80{ }^{\circ} \mathrm{C}$ (Figure 13 (b)). No significant change in spectra was found throughout the reaction, except for a small shift of the position of the main peak towards lower $q$ values. These results indicated that the resulting morphology corresponded to that present in the initial blend (before curing) but with an increased distance between nano-objects. As Figure 13(b) shows, all SAXS diagrams presented a slope of -4 in the high- $q$ region (Porod regime). This indicated that the dispersing objects had a smooth surface (Glatter and Kratky, 1982). The analysis showed that the spherical and cylindrical micelles were present in the initial blend, and were then frozen by the polymerization reaction. 

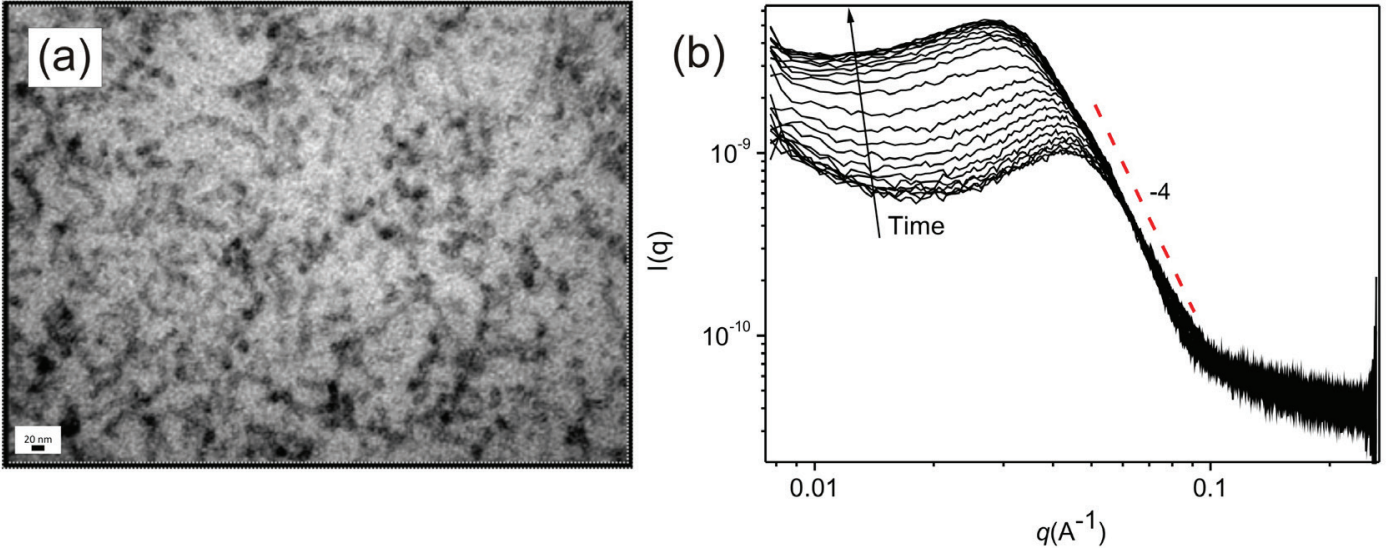

Figure 13. (a) TEM image of the epoxy blend with $10 \mathrm{wt} \%$ PEB- $b$-PEO fully cured at $80^{\circ} \mathrm{C}$. The specimen was stained with $\mathrm{RuO}_{4}$ prior to TEM observation. Scale bar $20 \mathrm{~nm}$. (b) In situ SAXS data obtained during curing at $80^{\circ} \mathrm{C}$ of the epoxy blend with 10 wt \% PEB-b-PEO. [Puig, J.; Ceolín, M.; Williams, R. J. J.; Schroeder, W. F.; Zucchi, I. A. Soft Matter 2017, 13 (40), 7341-7351] Reproduced by permission of The Royal Society of Chemistry.

\subsection{Photocuring at room temperature}

The blend containing $10 \mathrm{wt} \%$ PEB- $b$-PEO was also cured at room temperature by visible-light cationic photopolymerization. The mixture was activated for visible light irradiation by the addition of a photoinitiating system based on two components: $\mathrm{Ph}_{2} \mathrm{ISbF}_{6}(2 \mathrm{wt} \%)$ and CQ $(1 \mathrm{wt} \%)$. Under these conditions, a vitrification conversion of $0.62 \mathrm{was}$ reached after 4 hours of irradiation. The morphology of the resulting thermoset was characterized by transmission electron microscopy (TEM) and small-angle X-ray scattering (SAXS). Figure 14 (a) shows a TEM micrograph of the photo-cured sample stained with $\mathrm{RuO}_{4}$. Bilayer vesicles encapsulating the matrix can be observed, where the PEO corona is seen as darker lines surrounding the PEB core (lighter line). Mean vesicle diameter was $78.6 \pm 27.7 \mathrm{~nm}$, as determined from the analysis of several images.

To investigate whether the resulting vesicles were already present in the initial blend or formed during the reaction, in situ SAXS measurements were performed. Figure 14(b) shows the SAXS curves as a function of irradiation time. Contrary to the results obtained when curing was performed at $80^{\circ} \mathrm{C}$, in this case a continuous change in the SAXS pattern with reaction time was observed, indicating that the vesicular structure was the result of a polymerization-induced morphological transition. Information about the evolution of the micellar structure as a function of irradiation time was obtained from the slope of the low- $q$ region (Guinier regime) of the scattering profiles. At the beginning $(t=0$ min), the scattering profile showed a maximum at $q_{\max }=0.44 \mathrm{~nm}^{-1}$ indicating that the reactive blend was initially phase separated. This is in agreement with a nanostructured blend obtained through the self-assembly mechanism. As reaction progressed, the maximum reduced and finally disappeared at $75 \mathrm{~min}$ of reaction. At that time the low-q slope became equal to 0 , then it decreased to -1 close to $105 \mathrm{~min}$, and finally to -2 near $145 \mathrm{~min}$. According to theory, a low-q slope of 0 in the Guinier regime can be assigned to spheres, a slope of -1 to rigid rods and a slope of -2 to planar structures (like vesicles) (Glatter and Kratky, 1982). The results obtained from the Guinier analysis are in agreement with the commonly observed sequence of morphologies (i.e., sphere-to-cylinder-to-vesicle) derived from the increase in the segregation strength between the BCP and the epoxy matrix as polymerization progresses. SAXS data were analyzed using the SASfit software package in the $q$-region from 0.1 to $0.7 \mathrm{~nm}^{-1}$. The analysis showed that during the first $100 \mathrm{~min}$ of reaction, the system tended to reduce the total interfacial area by increasing micellar size while reducing the total number of micelles. As the reaction progressed, the spherical micelles converted into cylindrical micelles of smaller diameter, thus reducing system's total free energy. As polymerization progressed further, an analogous tendency to reduce the total free energy of the system forces a cylinder-to-vesicle transformation. The morphological transition (Sphere-Cylinder-Vesicle) was triggered by the change in the quality of the epoxy solvent during polymerization. The epoxy monomer, which acts as a selective solvent for the PEO block, produced a swollen "wet" PEO brush at the beginning of the reaction. During polymerization, the continuous increase in the size of epoxy oligomers decreased the miscibility of PEO through a reduction in the entropic contribution to the free energy of mixing. Under this circumstance, the polymerizing epoxy expelled the PEO brushes, creating conformational strains that induced a reduction of the local interfacial curvature. This is known as wet to dry brush transformation (Lipic et al. 1998). The appearance of vesicles was the response of the system to the conformational strain caused by the polymerization. 

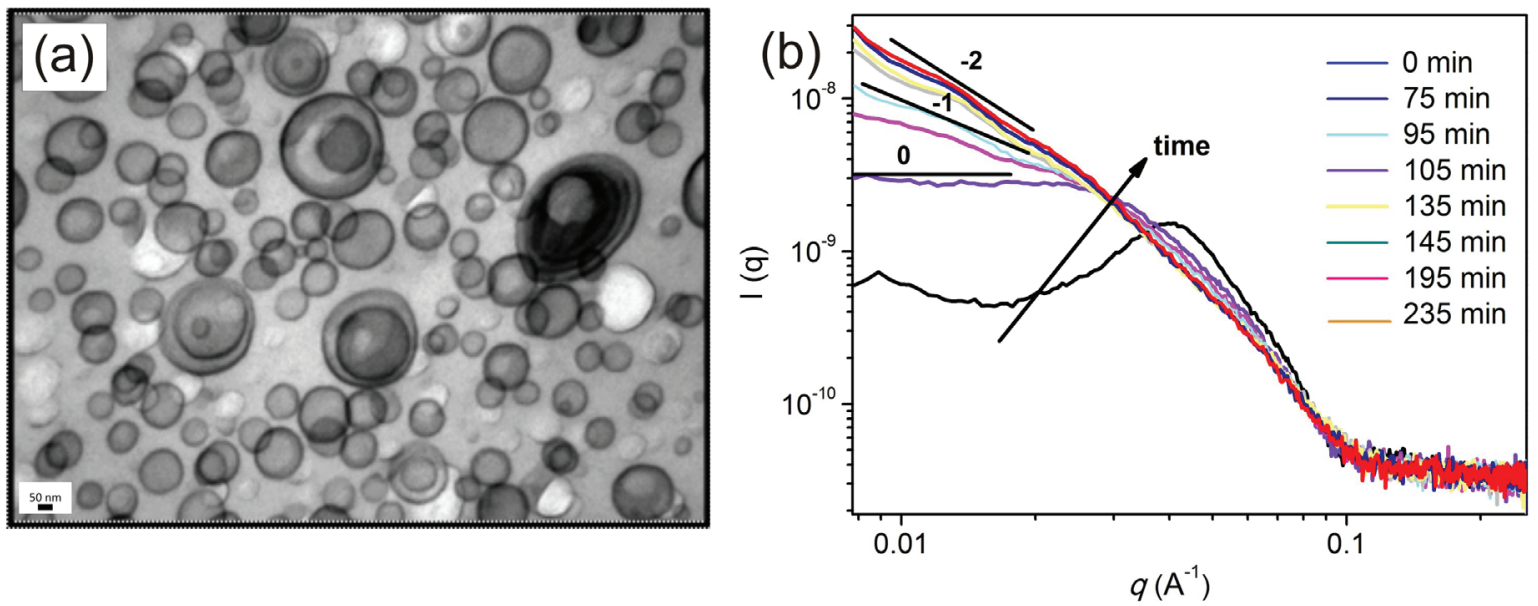

Figure 14. (a) TEM image of the epoxy blend with $10 \mathrm{wt} \%$ PEB- $b$-PEO photocured at room temperature. The specimen was stained with $\mathrm{RuO}_{4}$ prior to TEM observation. Scale bar $50 \mathrm{~nm}$. (b) In situ SAXS data obtained during photo-polymerization at room temperature of the epoxy blend with 10 wt \% PEB-b-PEO. [Puig, J.; Ceolín, M.; Williams, R. J. J.; Schroeder, W. F.; Zucchi, I. A. Soft Matter 2017, 13 (40), 7341-7351] Reproduced by permission of The Royal Society of Chemistry.

A question arises: why was the nanostructure obtained by curing at $80^{\circ} \mathrm{C}$ so different from that obtained at room temperature? The difference between the resulting morphologies (spheres vs. vesicles) was explained by comparing reaction kinetics. Figure 15 shows conversion vs. time curves for thermal cure at $80{ }^{\circ} \mathrm{C}$ and photocuring at room temperature. As it can be seen, both curves were identical until a conversion value of approximately 0.1 . After this conversion, reaction rate at $80{ }^{\circ} \mathrm{C}$ was markedly accelerated with respect to that at room temperature. It is evident that, under conditions of slow photopolymerization, the system could evolve from spheres to vesicles due to the high mobility of the matrix when transitions were triggered. On the contrary, when the cure was carried out at $80^{\circ} \mathrm{C}$, the initial spherical structures were kinetically trapped by diffusion constraints imposed by the fast polymerization of the matrix and the system could not evolve.

Once again, the critical role played by matrix mobility at the moment in which nanostructures evolved was evidenced. In this case, mobility could not be measured as a temperature gap between crystallization and $\mathrm{T}_{\mathrm{g}}$, since no crystallization of the immiscible block occurred. However, it was measured in terms of the diffusional constraints imposed by the conversion degree of the matrix at the moment of the morphological transition.

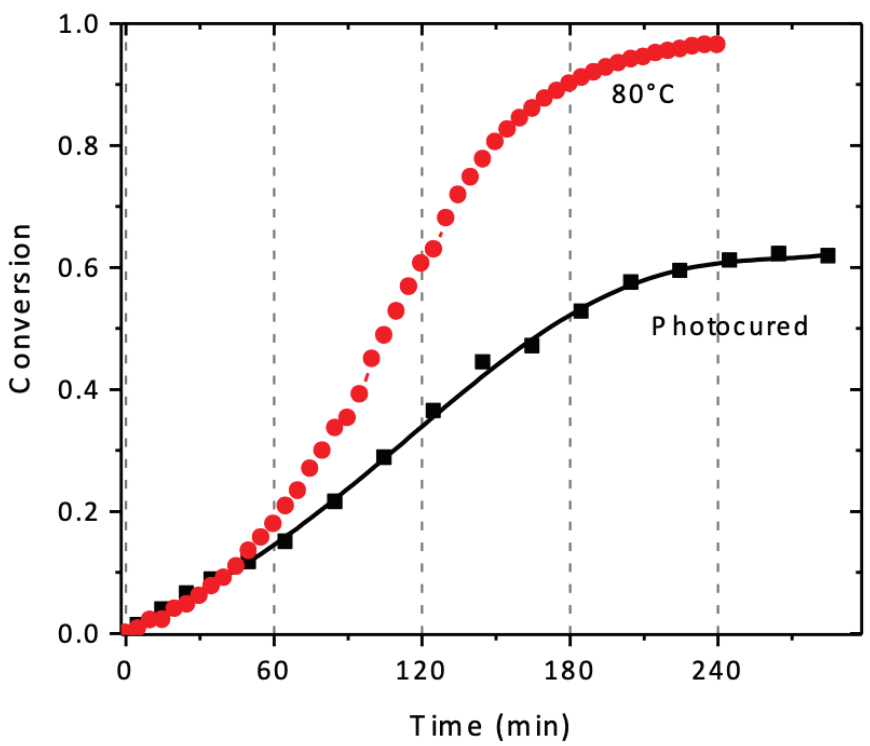

Figure 15. Conversion vs. time curves for the epoxy blend with $10 \mathrm{wt} \% \mathrm{PEB}-b-\mathrm{PEO}$, during thermal polymerization at $80{ }^{\circ} \mathrm{C}$ (circles) and photocuring at room temperature (squares). [Puig, J.; Ceolín, M.; Williams, R. J. J.; Schroeder, W. F.; Zucchi, I. A. Soft Matter 2017, 13 (40), 7341-7351] Reproduced by permission of The Royal Society of Chemistry. 


\section{Conclusions}

This review described different approaches reported to obtain novel block copolymer nanostructures dispersed in polymer matrices. It was demonstrated that the glass transition temperature $\left(\mathrm{T}_{\mathrm{g}}\right)$ of the matrix at the moment of the morphological transformation plays a decisive role determining the architecture of the nanostructure obtained.

Planar structures with precisely controlled length (ribbon-like micelles) can be obtained through a crystallization-driven self-assembly mechanism. In this case, micellar length can be tailored by regulating the temperature gap between the crystallization and the $T_{g}$ of the matrix. By this approach, structures ranging from discotic micelles to nanoribbons of very high aspect ratio can be obtained. In contrast, when crystallization is suppressed by matrix vitrification, amorphous rod-like micelles are obtained. On the other hand, when the core-forming block is an amorphous polymer, vesicles dispersed in an epoxy matrix can be generated through sphere-to-cylinder-to-vesicle morphological transition under slow photopolymerization conditions. It was demonstrated that matrix mobility, once again, plays a crucial role in allowing such transitions.

\section{Acknowledgments}

Financial support by ANPCyT, UNMdP and CONICET is gratefully acknowledged. I.A.Z., W.F.S. and J.P. are members of CONICET.

\section{References}

[1] J. Bang, U. Jeong, D.Y Ryu, T.P. Russell, and C.J. Hawker. Block Copolymer Nanolithography: Translation of Molecular Level Control to Nanoscale Patterns. Adv. Mater., 21: 4769-4792, 2009.

[2] C.E. Boott, E.M. Leitao, D.W Hayward, R.F. Laine, P. Mahou, G. Guerin, M.A. Winnik, R.M. Richardson,C.F. Kaminski,G.R. Whittell, G.R., et al.. Probing the Growth Kinetics for the Formation of Uniform 1D Block Copolymer Nanoparticles by Living Crystallization-Driven Self-Assembly. ACS Nano, 12: 8920-8933, 2018.

[3] L. Cao, I. Manners, and M.A. Winnik. Influence of the Interplay of Crystallization and Chain Stretching on Micellar Morphologies: Solution Self-Assembly of Coil-Crystalline Poly(isoprene-block-ferrocenylsilane). Macromolecules, 35: 8258-8260, 2002.

[4] J.J. Crassous, P. Schurtenberger, M. Ballauff, and A.M. Mihut. Design of block copolymer micelles via crystallization. Polymer 62: A1-A13, 2015.

[5] O.Glatter and O. Kratky. Small Angle X-ray Scattering, Academic Press, London, 1982.

[6] G. Guerin, G. Cambridge, M. Soleimani, S. Mastour Tehrani, I. Manners, and M.A. Winnik. Form Factor of Asymmetric Elongated Micelles: Playing with Russian Dolls Has Never Been so Informative. J. Phys. Chem. B, 118: 10740-10749, 2014.

[7] G. Guerin, P.A. Rupar, I. Manners and M.A. Winnik. Explosive dissolution and trapping of block copolymer seed crystallites. Nat. Commun., 9: 1158, 2018.

[8] Q. Guo, J.M. Dean, R.B. Grubbs, and F.S. Bates. Block copolymer modified novolac epoxy resin. J. Polym. Sci. Part B Polym. Phys., 41: 1994-2003, 2003a.

[9] Q. Guo, R. Thomann, W. Gronski, R. Staneva, R. Ivanova, and B. Stühn. Nanostructures, Semicrytalline Morphology, and Nanoscale Confinement Effect on the Crystallization Kinetics in Self-Organized Block Copolymer/Thermoset Blends. Macromolecules, 36: 3635-3645, $2003 \mathrm{~b}$.

[10] R.C. Hayward and D.J. Pochan. Tailored Assemblies of Block Copolymers in Solution: It Is All about the Process. Macromolecules, 43: 3577-3584, 2010.

[11] W.-N. He and J-T. Xu. Crystallization assisted self-assembly of semicrystalline block copolymers. Prog. Polym. Sci., 37: 1350-1400, 2012a.

[12] W.-N. He, B. Zhou, J.-T. Xu, B-Y. Du and Z.-Q. Fan. Two Growth Modes of Semicrystalline Cylindrical Poly( $\varepsilon-c a p r o-$ lactone)-b-poly(ethylene oxide) Micelles. Macromolecules, 45: 9768-9778, 2012b. 
[13] A.B. Leonardi, I.A. Zucchi and R.J.J. Williams. Generation of large and locally aligned wormlike micelles in block copolymer/epoxy blends. Eur. Polym. J., 65: 202-208, 2015.

[14] P.M Lipic, F.S. Bates and M.A. Hillmyer. Nanostructured Thermosets from Self-Assembled Amphiphilic Block Copolymer/Epoxy Resin Mixtures. J. Am. Chem. Soc. ,120: 8963-8970, 1998.

[15] Y. Mai and A. Eisenberg. Self-assembly of block copolymers. Chem. Soc. Rev. ,41 :5969-5985, 2012.

[16] F. Meng, S. Zheng, H. Li, Q. Liang and T. Liu. Formation of Ordered Nanostructures in Epoxy Thermosets: A Mechanism of Reaction-Induced Microphase Separation. Macromolecules, 39: 5072-5080, $2006 \mathrm{a}$.

[17] F. Meng, S. Zheng and T. Liu. Epoxy resin containing poly(ethylene oxide)-block-poly( $\varepsilon$-caprolactone) diblock copolymer: Effect of curing agents on nanostructures. Polymer, 47: 7590-7600, 2006b.

[18] R.M. Michell and A. J. Müller. Confined crystallization of polymeric materials. Prog. Polym. Sci., 54-55: 183-213, 2016.

[19] A.M. Mihut, M. Drechsler, M. Möller and M. Ballauff. Sphere-to-Rod Transition of Micelles formed by the Semicrystalline Polybutadiene-block-Poly(ethylene oxide) Block Copolymer in a Selective Solvent. Macromol. Rapid Commun., 31: 449-453, 2010.

[20] Ú.M. Montoya Rojo, C.C. Riccardi, M.D. Ninago, A.E. Ciolino, M.A. Villar, M. Ceolín, I.A. Zucchi and W.F. Schroeder. Photopolymerization-assisted self-assembly as a strategy to obtain a dispersion of very high aspect ratio nanostructures in a polystyrene matrix. Eur. Polym. J., 112: 704-713, 2019.

[21] M. Nakano, K. Matsumoto, H. Matsuoka and H. Yamaoka. Characterization of Micellization Behavior of Amphiphilic Polymer Having Octadecyl Group by Small-Angle X-ray and Neutron Scattering. Macromolecules, 32: 4023-4029, 1999.

[22] A.N. Parikh, S.D. Gillmor, J.D. Beers, K.M. Beardmore, R.W. Cutts and B.I. Swanson. Characterization of Chain Molecular Assemblies in Long-Chain, Layered Silver Thiolates: A Joint Infrared Spectroscopy and X-ray Diffraction Study. J. Phys. Chem. B , 103: 2850-2861, 1999.

[23] J. Puig, I.A. Zucchi, M. Ceolín, W.F. Schroeder and R.J.J. Williams. Evolution of morphologies of a PE-b-PEO block copolymer in an epoxy solvent induced by polymerization followed by crystallization-driven self-assembly of PE blocks during cooling. RSC Adv., 6: 34903-34912, 2016.

[24] J. Puig, M. Ceolín, R.J.J Williams, W.F. Schroeder and I.A. Zucchi. Controlling the generation of bilayer and multilayer vesicles in block copolymer/epoxy blends by a slow photopolymerization process. Soft Matter, 13: 7341-7351, 2017.

[25] D. Richter, D. Schneiders, M. Monkenbusch, L. Willner, L.F. Fetters, J.S. Huang, M. Lin, K. Mortensen and B. Farago. Polymer Aggregates with Crystalline Cores: The System Polyethylene-Poly(ethylenepropylene). Macromolecules, 30: 1053-1068, 1997.

[26] R.N. Schmarsow, M. Ceolín, I.A. Zucchi and W.F. Schroeder. Core-crystalline nanoribbons of controlled length via diffusion-limited colloid aggregation. Soft Matter, 15:4751-4760, 2019.

[27] L. Shen, H. Wang, G. Guerin, C. Wu, I. Manners and M.A. Winnik. A Micellar Sphere-to-Cylinder Transition of Poly(ferrocenyldimethylsilane-b-2-vinylpyridine) in a Selective Solvent Driven by Crystallization. Macromolecules, 41: 4380-4389, 2008.

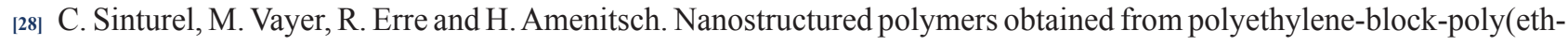
ylene oxide) block copolymer in unsaturated polyester. Macromolecules, 40: 2532-2538, 2007.

[29] C. Sinturel, M. Vayer, R. Erre and H. Amenitsch. Thermal induced mobility of self-assembled platelets of polyethylene-block-poly(ethylene oxide) in liquid precursors of unsaturated polyester thermoset. Eur. Polym. J., 45: 2505$2512,2009$.

[30] A. Tercjak, M. Larrañaga, M.D. Martin and I. Mondragon. Thermallyreversible nanostructured thermosetting blends modified with poly(ethylene-b-ethyleneoxide) diblock copolymer. J. Therm. Anal. Calorim., 86: 663-667, 2006. 
[31] S.Tiptipakorn, N. Keungputpong, S. Phothiphiphit and S. Rimdusit. Effects of polycaprolactone molecular weights on thermal and mechanical properties of polybenzoxazine. J. Appl. Polym. Sci. 132 (11pp), 2015.

[32] Trent, J.S., Scheinbeim, J.I., and Couchman, P.R. (1983). Ruthenium tetraoxide staining of polymers for electron microscopy. Macromolecules, 16:589-598, 1983.

[33] Wang, X., Guerin, G., Wang, H., Wang, Y., Manners, I., and Winnik, M.A. Cylindrical Block Copolymer Micelles and Co-Micelles of Controlled Length and Architecture. Science, 317 :644-647, 2007.

[34] J. Wu, Y.S. Thio and F.S. Bates. Structure and properties of PBO-PEO diblock copolymer modified epoxy. J. Polym. Sci. Part B Polym. Phys., 43: 1950-1965, 2005.

[35] L.Yin, T.P. Lodge and M.A. Hillmyer. A Stepwise "Micellization-Crystallization" Route to Oblate Ellipsoidal, Cylindrical, and Bilayer Micelles with Polyethylene Cores in Water. Macromolecules, 45: 9460-9467, 2012.

[36] C.Zhang, L. Li and S. Zheng. Formation and Confined Crystallization of Polyethylene Nanophases in Epoxy Thermosets. Macromolecules, 46, 2740-2753, 2013.

[37] I.A. Zucchi and W.F. Schroeder. Nanoribbons with semicrystalline core dispersed in a visible-light photopolymerized epoxy network. Polymer, 56, 300-308, 2015.

\section{Bios}

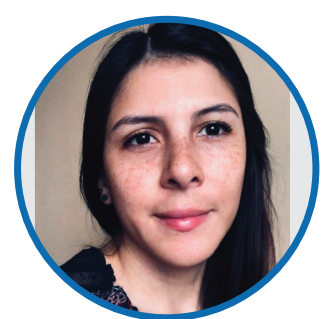

Jessica Gutiérrez González

Jessica Gutiérrez González

received her B.Sc. degree in

Chemistry from University of

Quindío, Colombia. From 2013

to 2016 , she participated in biological sciences projects at the

National University of La Plata (UNMdP, Argentina). She is currently a Ph. D student in Material Sciences at the UNMdP. Her main research interests include the study and characterization of nanostructured materials obtained by the self-assembly of semi-crystalline block copolymers.

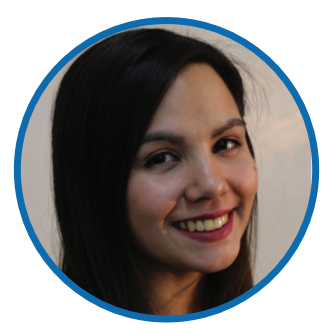

\section{Ruth N. Schmarsow}

Ruth N. Schmarsow received her B.Sc. degree in Chemistry from the National University of Mar del Plata (UNMdP, Argentina) in 2017. She is a teaching associate at the Department of Chemistry of the UNMdP. She is currently a Ph.D student in Material Sciences at the School of Engineering of the UNMdP. Her main research interest is the synthesis and characterization of functional materials obtained by semi-crystalline block copolymer self-assembly in different polymer matrices.

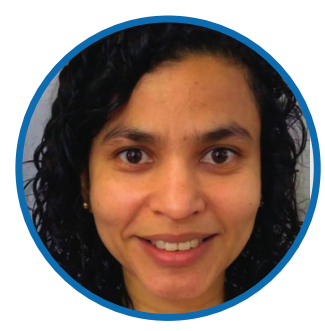

\section{Úrsula Montoya Rojo}

Úrsula Montoya Rojo is an assistant professor at the School of Engineering of the University of Buenos Aires (UBA, Argentina). She has a degree on Agroindustrial Engineering from University Pontificia Bolivariana (Colombia). She received her $\mathrm{PhD}$ in Materials Science from the National University of Mar del Plata (UNMdP, Argentina) in 2017. She is currently a postdoctoral fellow at the Biotechnology and Biosynthesis Group (ITPN-UBA-CONICET, Argentina). She has been active in the field of nanostructures of biopolymers based on vegetable and bacterial cellulose nanofibers as well as on self-assembly as a strategy to obtain nanostructures in different polymeric networks.

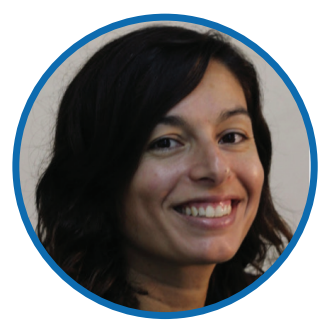

\section{Julieta Puig}

Julieta Puig obtained her B.Sc. in Chemistry (2009) and PhD in Materials Science (2014) from the $\mathrm{Na}$ tional University of Mar del Plata (UNMdP, Argentina). Her PhD focused on different tailor-made polymeric matrices and their modification using several types of hydrophobic modifiers for the development of functional materials with technological properties. For her postdoc, she worked in the generation of novel nanostructures of block copolymers in an epoxy monomer via photo- and thermal polymerization. She is currently working as an Assistant Researcher at INTEMA-CONICET. Her main research interest is the preparation of functional nanostructured materials with special optical and thermal properties for the development of Smart Windows. 


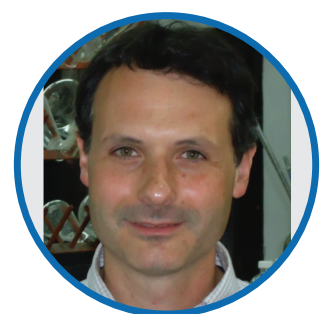

\section{Walter F. Schroeder}

Walter F. Schroeder obtained his B.Sc and Ph.D. degrees at University of Mar del Plata (UNMdP, Argentina). He was a postdoctoral fellow in the Department of Chemistry at the University of Toronto (Canada), where he studied polymer diffusion by Fluorescence Resonance Energy Transfer (FRET). He is currently a scientific researcher for CONICET and a professor at the Department of Chemical Engineering of the UNMdP. His main research interest is the study of the kinetic and thermodynamic aspects involved in the formation of nanostructured materials obtained by self-assembly of block copolymers in polymer matrices.

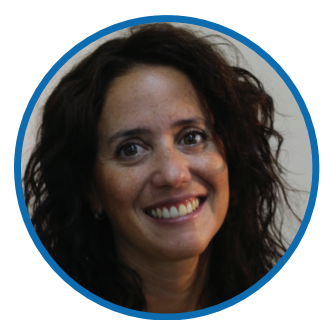

lleana A. Zucchi

Ileana A. Zucchi obtained her B.Sc. and Ph.D. degrees from the National University of Mar del Plata (UNMdP, Argentina). She was a postdoctoral fellow at the Institute for Microelectronics and Microsystems of the National Research Council of Italy, studying the generation of block copolymer templates for advanced nanolithography applications. Since 2008, she is a researcher scientist for CONICET and teaching assistant at the Department of Chemical Engineering of the UNMdP. Her research focuses on the development of functional materials through the self-assembly of block copolymers. 